\title{
Design and accuracy analysis of multi-level state estimation based on smart metering infrastructure
}

\author{
Marco Pau Member, IEEE, Edoardo Patti Member, IEEE, Luca Barbierato Student Member, IEEE, \\ Abouzar Estebsari Member, IEEE, Enrico Pons Member, IEEE, Ferdinanda Ponci Senior Member, IEEE, \\ Antonello Monti Senior Member, IEEE
}

\begin{abstract}
While the first aim of smart meters is to provide energy readings for billing purposes, the availability of these measurements could open new opportunities for the management of future distribution grids. This paper presents a multi-level state estimator that exploits smart meter measurements for monitoring both low and medium voltage grids. The goal of the paper is to present an architecture able to efficiently integrate smart meter measurements and to show the accuracy performance achievable if the use of real-time smart meter measurements for state estimation purposes were enabled. The design of the state estimator applies the uncertainty propagation theory for the integration of the data at the different hierarchical levels. The coordination of the estimation levels is realized through a cloud-based infrastructure, which also provides the interface to auxiliary functions and the access to the estimation results for other distribution grid management applications. A mathematical analysis is performed to characterize the estimation algorithm in terms of accuracy and to show the performance achievable at the different levels of the distribution grid when using the smart meter data. Simulations are presented, which validate the analytical results and demonstrate the operation of the multi-level estimator in coordination with the cloud-based platform.
\end{abstract}

Keywords-State Estimation, Distribution System Automation, Distributed Estimation, Smart Meters, Distribution Grid Monitoring, Measurement Uncertainty, Cloud Platform.

\section{INTRODUCTION}

Electric distribution grids are evolving into complex systems where many different resources, like distributed generation, storage systems and flexible loads, have to be coordinated to achieve the efficient and secure operation of the network [1]. The smart control of these heterogeneous resources requires the development of new applications for the management of the grid at both Medium Voltage (MV) and Low Voltage (LV) level [2]. Most of the functions of the Distribution Management System (DMS) need to know the operating conditions of the network to operate [3]. This information is provided by the Distribution System State Estimation (DSSE), which is thus crucial to enable the intelligent functions required for the control of future distribution grids.

M. Pau, F. Ponci and A. Monti are with the Institute for Automation of Complex Power Systems, RWTH Aachen University, Aachen, Germany. Email: \{mpau, fponci, amonti\}@eonerc.rwth-aachen.de

E. Patti, L. Barbierato are with the Energy Center Lab, Department of Control and Computer Engineering, Politecnico di Torino, Turin, Italy. Email: \{edoardo.patti, luca.barbierato\}@ polito.it

A. Estebsari and E. Pons are with the Department of Energy, Politecnico di Torino, Turin, Italy. Email: \{abouzar.estebsari, enrico.pons\}@polito.it
In spite of the large research efforts to design State Estimation (SE) algorithms tailored to distribution grids [4]-[8], some important issues still hinder the deployment of DSSE in real networks [9]. The main obstacle is the scarcity of measurements on the field at distribution level, which prevents the effective operation of DSSE. Common solutions rely on pseudo-measurements, namely forecasts of the power generation or consumption at the different nodes of the network [10], [11]. While this solution allows reaching the observability of the system and makes DSSE possible, the achievable accuracy performance is significantly affected [12]. Due to these problems, the distribution systems need an upgrade of the measurement system in order to evolve into smart grids.

Several works have tackled the problem of meter placement in distribution systems [13]-[16]. One of the solutions recently investigated is the use of smart meters at customer premises as input for the DSSE. While these devices are mainly intended for billing purposes, their measurements can actually be a precious source of information for the management of distribution grids. Relying on this idea, different papers proposed the use of smart meters for DSSE. In [17], smart meters placed in strategical points are used to enhance the observability of the distribution grid. Similarly, the technique proposed in [18] relies upon the placement of smart meters in few selected locations to enable the grid observability. In [19] and [20], the main idea is to aggregate the information given by the smart meters to enhance the knowledge of the power injections at the MV/LV substations of the MV grid. Other works aim instead at including smart meter measurements directly on LV grid estimators [21], [22]. Field trials showing the use of smart meters for LV grid monitoring have been also presented recently (see for example [23] and [24]). All these papers, however, use the smart meter measurements only for one level of the distribution grid, not allowing the full monitoring of the distribution network, at both LV and MV level, based on the use of smart meters.

Differently from the above-mentioned works, this paper overcomes this limitation and presents a state estimator that uses smart meter measurements to monitor both LV and MV grids. A multi-level architecture is proposed to this purpose, where the SE problem is decomposed into concentrator, LV grid and MV grid SE levels. Such a solution allows distributing computation, memory and communication requirements, thus facilitating the DSSE execution and coping with the challenge of deploying DSSE in large distribution networks. In [25], a first version of the estimator was presented, but its application 
was limited only to the LV grid. In this work, the DSSE algorithm has been refined to allow also the monitoring of MV grids, thus extending the benefits of the use of smart meter measurements to the upper level of the distribution system. A similar concept is presented also in [26], where LV grid estimations are used in input to the MV estimator. However this paper presents more in detail how to coordinate the hierarchical levels of the SE architecture, referring to the use of the uncertainty propagation law for the accurate integration of the results achieved at the different SE levels. The main contributions of this work, which represent technical novelties with respect to the state of the art literature, can be listed as follows.

- The details on how to model the MV/LV transformer to fully exploit the LV grid results into the MV grid level SE are provided.

- The coordination of the SE levels using the uncertainty propagation theory is presented: the goal here is to maximize the accuracy performance and to make possible the mathematical evaluation of such performance.

- The accuracy performance achievable at the different levels of the DSSE architecture is presented. To this purpose, the analytical expressions needed to compute the uncertainty of voltages and powers as a function of the number of smart meters and of their accuracy characteristics have been derived; numerical simulations are then used to validate the mathematical findings.

Together with these main contributions, to give an overall view of the conceived architecture, a cloud-based infrastructure used to interconnect the different DSSE levels is also presented [27]. This infrastructure also allows interfacing other DSSE auxiliary functions and storing the DSSE results for their use in other distribution system management functions.

The remainder of this paper is organized as follows. In Section II, the design of the multi-level DSSE algorithm is described, while Section III presents the cloud-based infrastructure used to coordinate the hierarchical levels of the estimator. In Section IV, the mathematical analysis developed to assess the accuracy performance of the smart meter based DSSE is presented. Simulations validating the analytical findings and showing the operation of the multi-level DSSE algorithm in the proposed cloud architecture are discussed in Section V. Finally, Section VI provides the final remarks of the paper.

\section{Multi-Level State Estimation}

\section{A. Multi-level architecture}

The DSSE scheme presented in this paper is composed of three hierarchical levels conceived to distribute the computational requirements for SE and to fully exploit smart meter measurements for monitoring both LV and MV grid. Starting from the lowest level of the hierarchy, the proposed estimator relies upon: concentrator SEs; LV grid SEs; MV grid SE (Fig. 1). To remark the presence of the different hierarchical levels, the architecture will be referred to as multi-level in the following.

In a smart metering infrastructure, the concentrators aggregate the smart meter readings coming from different customers,

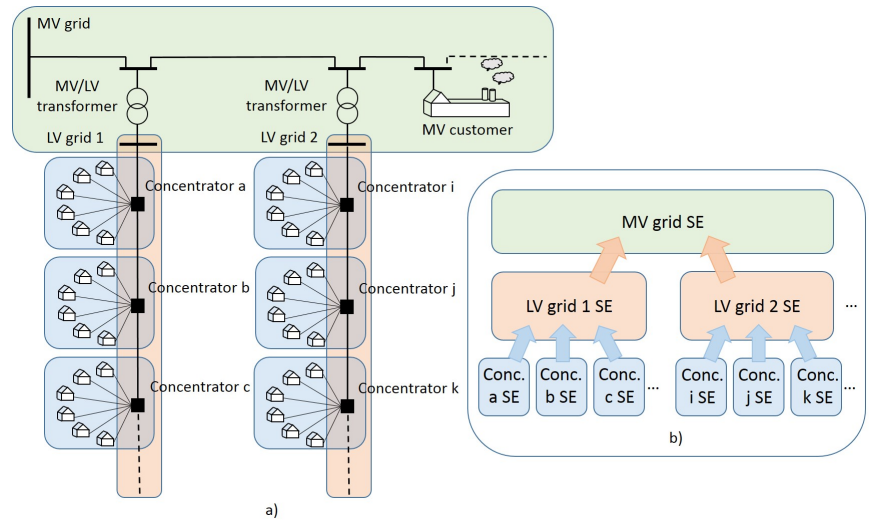

Fig. 1. Multi-level SE architecture: a) partition of the distribution grid; b) hierarchical structure of the architecture.

translate them (if needed) into the data format used at the following acquisition stage, and forward them periodically with a chosen reporting rate. To perform these tasks, concentrators are equipped with computational capabilities, hence, the idea, already anticipated in [25], is to use these computational resources to compute a first stage (concentrator SE) of the SE process to estimate the voltages and the power injections seen the LV feeder buses. As depicted in the simplified schema in Fig. 1a, thanks to the radial topology of LV grids and the resulting configuration of feeders and laterals, it is possible to place the concentrators in the LV feeder buses to have the aggregation of all the smart meters connected to such bus. Using this criterion, each concentrator SE can monitor the laterals of the LV grid departing from the associated feeder bus and estimate, as a result, the voltage and the equivalent power injection seen from such node on the LV feeder (see the area associated to each concentrator in Fig. 1a).

The second stage (LV grid SE) of the multi-level SE process estimates the state of the LV grid by using as input the SE results on the LV feeder buses provided by the concentrator level estimators. This SE process allows the monitoring of the operating conditions in the LV feeders, which can be also used to trigger possible functions designed for the management of the LV grid. Similarly to the previous stage, the LV grid monitoring estimates the voltage state and the overall power injection resulting at the MV/LV substation. This, in turn, enables the application of an upper level SE on the MV network (MV grid SE), which enables the full monitoring of the MV grid without installing additional meters at MV level. In fact, if a full coverage of smart meters is available on the distribution grid, each MV/LV substation would have an equivalent voltage and power injection input given by the LV grid SE, while the measurement information at the nodes directly connected to MV customers could be obtained through their smart meters. This guarantees the observability of the MV grid even in a worst-case scenario where no other measurement instrumentation is present at MV level and without the need to use inaccurate pseudo-measurements.

The multi-level architecture (Fig. 1b) presented here is developed only for the distribution level of the electric system, 
but it can be expanded to fit the hierarchical structure theorized in [28] for a comprehensive smart grid SE including both distribution and transmission systems. The presented DSSE scheme thus allows addressing the following challenges.

- Smart meter measurements are fully exploited to obtain situational awareness on the whole distribution grid (both LV and MV level), enabling the development of advanced distribution management functions. The use of smart meters allow monitoring the whole distribution grids without the need for large investments to deploy additional measurement instruments.

- As it will be shown in Section IV, the use of smart meters together with the proposed coordination framework allow having a very accurate tracking of the grid operating conditions, differently from what achievable by adopting pseudo-measurements.

- SE can be performed in parallel among different LV grids and among different concentrators within the same LV grid; this allows splitting the SE problem, distributing computational and memory requirements and reducing the required execution times: this is a key aspect given the very large size of the distribution grids.

- Concentrators send only SE results in real-time at the LV feeder buses and not the whole set of smart meter measurements, with consequent reduction of the realtime communication requirements.

\section{B. State Estimation model}

The SE algorithms implemented at the different stages of the multi-level architecture are all based on the well-known Weighted Least Squares (WLS) approach. The goal of the WLS technique is to minimize the following weighted sum (see [29] for more details):

$$
J(x)=\sum_{i=1}^{M} w_{i}\left(z_{i}-h_{i}(\mathbf{x})\right)^{2}
$$

where $z_{i}$ is the $i$-th available measurement; $h_{i}(\mathbf{x})$ is the associated measurement function expressing the measured quantity in terms of the state variables used in the vector $\mathbf{x} ; w_{i}$ is a weight used to scale the importance of the measurements according to their accuracy (inverse of the measurement variance $\sigma_{i}^{2}$ ). Converting the objective function in (1) in matrix form and applying the Gauss-Newton method to pursue the minimization, the final state vector is obtained through the iterative solution of the following equation system:

$$
\mathbf{G} \Delta \mathbf{x}=\mathbf{H}_{\mathbf{z}}{ }^{T} \mathbf{W}_{\mathbf{z}}\left[\mathbf{z}-\mathbf{h}_{\mathbf{z}}(\mathbf{x})\right]
$$

where $\mathbf{z}$ and $\mathbf{h}_{\mathbf{z}}(\mathbf{x})$ are the vectors containing the $z_{i}$ and $h_{i}(\mathbf{x})$ values; $\mathbf{W}_{\mathbf{z}}$ is the weighting matrix having the $w_{i}$ terms on the diagonal; $\mathbf{H}_{\mathbf{z}}$ is the Jacobian matrix associated to the measurement functions $h_{i}(\mathbf{x}) ; \mathbf{G}=\mathbf{H}_{\mathbf{z}}{ }^{T} \mathbf{W}_{\mathbf{z}} \mathbf{H}_{\mathbf{z}}$ is the socalled Gain matrix; $\Delta \mathrm{x}$ is the updating vector to be summed to the state vector $\mathbf{x}$ at each iteration to update its result. The iterative procedure stops after the largest element in $\Delta \mathrm{x}$ (in absolute value) is smaller than a chosen threshold.

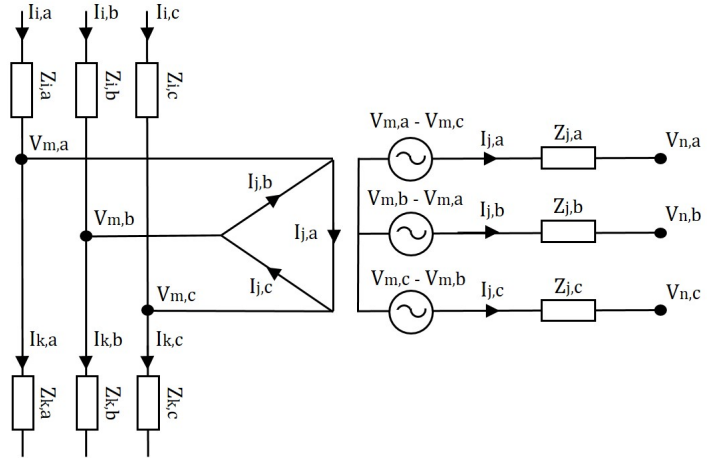

Fig. 2. MV/LV substation model for the MV grid SE.

The WLS approach is often used in the SE context because under the hypothesis of Gaussian distribution of the measurement uncertainties it corresponds to the maximum likelihood estimator [29]. Another important advantage offered by the WLS formulation is the possibility to compute the corresponding uncertainty associated to the estimated state variables: this can be calculated as the inverse of the Gain matrix $\Sigma_{\mathbf{x}}=\mathbf{G}^{-1}$. Such an aspect is of paramount importance for the application of a hierarchical SE architecture; in fact, calling with $\mathbf{y}=\mathbf{h}_{\mathbf{y}}(\mathbf{x})$ the vector of electrical quantities to be provided as input to the upper level SE, it is possible to compute their uncertainty $\boldsymbol{\Sigma}_{\mathbf{y}}$ by applying the law of propagation of the uncertainties:

$$
\boldsymbol{\Sigma}_{\mathbf{y}}=\mathbf{H}_{\mathbf{y}} \boldsymbol{\Sigma}_{\mathbf{x}} \mathbf{H}_{\mathbf{y}}{ }^{T}
$$

where $\mathbf{H}_{\mathbf{y}}$ is the Jacobian associated to the measurement functions $\mathbf{h}_{\mathbf{y}}(\mathbf{x})$ (which express the quantities in $\mathbf{y}$ in terms of the SE state variables in $\mathbf{x}$ ). The diagonal terms in $\boldsymbol{\Sigma}_{\mathbf{y}}$ are the resulting variances of the quantities in $\mathbf{y}$ and can be thus used for the accurate definition of the weights to be introduced in the WLS procedure at the upper levels of the SE hierarchy.

\section{State Estimation algorithm}

The SE algorithm implemented in all the three stages of the architecture is based on the three-phase unbalanced formulation presented in [8]. The state vector $\mathrm{x}$ is composed of the voltage magnitude at a reference node and the currents at all the branches of the grid, expressed in rectangular form. It is worth noting that, as proved in [30], other WLS formulations based on different state variables also provide the same estimation results with same accuracy. As a consequence, the choice of the SE algorithm could be also different and the choice shown here is not a constraint for the proposed architecture.

The design of the algorithm at the concentrator and the LV grid level is straightforward and simply follows the steps described in [8]. At MV level, instead, the presence of the MV/LV transformers has to be considered and suitably modelled. Since most of the distribution grids are characterized by MV/LV transformers with a delta-grounded star connection, this configuration has been considered here for the design of the algorithm. Its modelling follows the scheme in Fig. 2 (note 
that the transformer ratio can be neglected when working with per unit values, as it usually occurs in SE). In particular, the branch currents at the secondary side of the transformer are included among the state variables $\mathrm{x}$ of the MV SE algorithm (branch $j$ in Fig. 2). Given these additional state variables, and referring to the scheme in Fig. 2, it is possible to see that:

- according to [8], power injections are converted at each SE iteration in equivalent current measurements; thus, the power injection seen from the MV/LV substation (node $n$ ) is converted into the real and imaginary currents $i_{i n j, n, \phi}^{r e}$ and $i_{i n j, n, \phi}^{i m}$ and for each phase $\phi=a, b, c$ their measurement functions are:

$$
\begin{aligned}
& h_{i i_{i n j, n, \phi}^{r e}}=i_{j, \phi}^{r e} \\
& h_{i i_{i n j, n, \phi}^{i m}}=i_{j, \phi}^{i m}
\end{aligned}
$$

- due to the delta connection at the primary side of the transformer, the zero current injection at node $m$ creates a coupling among the different phases of the system; as an example, for the current injection $i_{i n j, m, a}^{r e}$ at phase $a$, the corresponding measurement function is:

$$
h_{i_{i n j, m, a}^{r e}}=i_{i, a}^{r e}-i_{k, a}^{r e}-i_{j, a}^{r e}+i_{j, b}^{r e}
$$

- the voltage magnitude measurement at the MV/LV substation (node $n$ ) also introduces a coupling among the phases; for example, for the voltage $V_{n, a}$ at phase $a$, the resulting measurement function is (see [8] for more details on its computation):

$$
\begin{aligned}
h_{V_{n, a}} & =V_{1, a} \cdot \cos \left(\theta_{1, a}-\theta_{n, a}\right)-V_{1, c} \cdot \cos \left(\theta_{1, c}-\theta_{n, a}\right)- \\
& \sum_{p \in \Gamma_{1-n}}\left(r_{p, a} i_{p, a}^{r e}-x_{p, a} i_{p, a}^{i m}\right) \cdot \cos \left(\theta_{n, a}\right)- \\
& \sum_{p \in \Gamma_{1-n}}\left(r_{p, a} i_{p, a}^{i m}+x_{p, a} i_{p, a}^{r e}\right) \cdot \sin \left(\theta_{n, a}\right)+ \\
& \sum_{p \in \Gamma_{1-m}}\left(r_{p, c} i_{p, c}^{r e}-x_{p, c} i_{p, c}^{i m}\right) \cdot \cos \left(\theta_{n, a}\right)+ \\
& \sum_{p \in \Gamma_{1-m}}\left(r_{p, c} i_{p, c}^{i m}+x_{p, c} i_{p, c}^{r e}\right) \cdot \sin \left(\theta_{n, a}\right)
\end{aligned}
$$

where $\theta_{s, \phi}$ is the voltage angle at bus $s$ for phase $\phi, r_{p, \phi}$ and $x_{p, \phi}$ are the resistance and reactance, respectively, associated to the phase $\phi$ of branch $p$, and $\Gamma_{1-s}$ is the path between the starting reference node of the grid and the generic node $s$.

\section{Software Smart Metering Architecture}

\section{A. Software platform}

Monitoring and controlling the electricity consumption at the customers level to improve efficiency is an important feature of future smart grids [31], [32]. The deployed smart metering infrastructure plays a key role to achieve this objective. Indeed, it needs to manage millions of internet-connected devices (e.g. smart meters) in a secure, reliable and scalable way. To enable this, utilities need distributed data-centres. In

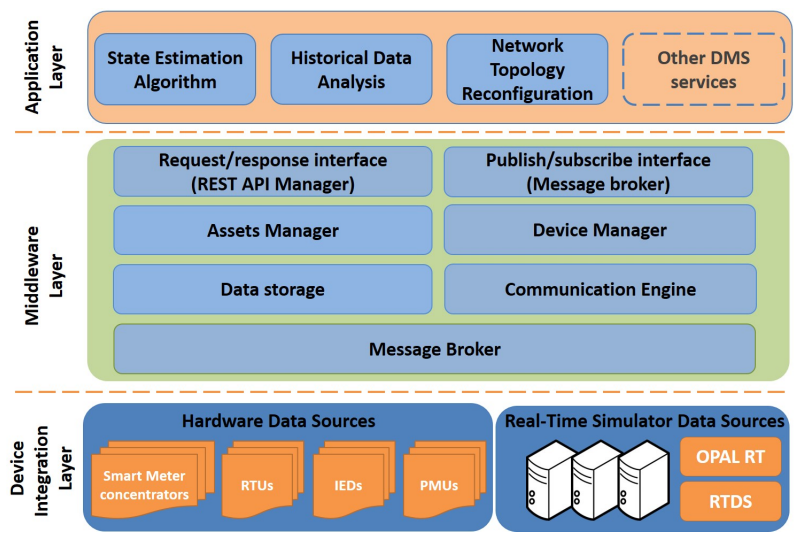

Fig. 3. Architectural schema of the cloud platform

this respect, cloud computing is envisaged to play a key role in future smart grids [33]. As highlighted in [33], sharing information among different actors and services is also a crucial issue in the smart grid scenario. This data sharing can enable new business models and novel services to manage the power distribution network, fostering new actors to participate in a fast-evolving distributed marketplace [34].

The smart metering infrastructure used in this paper aims at providing the above features [27]. In particular, it allows the interoperability among: i) different services and ii) heterogeneous devices to retrieve measurement information. Furthermore, it is capable of handling and post-processing huge amounts of information. The cloud platform here presented is, therefore, not only the key enabler to coordinate the different levels of the SE architecture, but also gives the possibility to interface SE with other possible services for the management of the distribution grid. As shown in Figure 3, the platform is a three layer architecture with: i) a Device Integration Layer, ii) a Middleware Layer and iii) an Application Layer.

The cloud platform is able to deal with heterogeneous devices using different communication protocols. This means that, if different measurements are available in the grid (using different protocols), these can be also exploited into the DSSE algorithm. To enable interoperability among different technologies, the Device Integration Layer exploits Device Integration Adapters (DIA) to abstract the hardware datasources through a methodology described in [35]. The DIA is a software component that converts the measurements coming from the devices to the JSON data format used within the cloud platform and sends them to the cloud infrastructure exploiting the MQTT protocol [36].

The Middleware Layer is composed of different software components acting together to: allow communication with DIAs; receive, check and store measurements; provide access to data, devices, assets and maintenance operations. The Message Broker provides an asynchronous communication through MQTT, which is a publish/subscribe protocol to send data in (near-) real-time. This communication paradigm removes the interdependencies between producer and consumer of information increasing the scalability of the whole infrastructure [34].

The Message Broker and the Communication Engine allow 
a bi-directional communication between the software platform and smart devices (i.e. smart meters and/or smart appliances). In addition, the Communication Engine is responsible for checking messages integrity, managing traffic spikes and collecting the incoming measurements into a Data Storage composed of different non-relational databases designed for Big Data management. This approach provides scalability and clusterization keeping the cloud infrastructure independent from the low-level database management systems. Finally, the REST API Interface Manager provides REST web services to access information and manage entities in the infrastructure through a request/response communication paradigm. All REST web services need authentications to allow the users to perform operations. Thanks to this module, the platform provides developers with uniform interfaces to access stored information allowing them the development of new services based on the available data.

The Application Layer is the highest layer of the platform. It provides tools and interfaces to design distributed applications and services for smart grid management and user awareness. The multi-level state estimation algorithm presented in this paper is an example of such services. In general, applications can exchange data with the cloud platform using two different communication paradigms. The first option is to consider a publish/subscribe approach using the MQTT protocol: in this case applications can publish new information and subscribe to receive data in (near-) real-time. This option is particularly suited for applications that have to react as soon as new information is delivered or a new event is triggered. The second option is to use a request/response communication via REST. In this case applications can write, modify or access data available in the cloud whenever it is needed regardless of the moment in which those data were produced or generated. More details on the features and performance of the used cloud platform can be found in [27].

\section{B. Data and communication flows for the multi-level SE}

Figure 4 shows a schematic of the communication flows needed for the coordination of the multi-level SE algorithm in the used cloud platform. As explained in Section II-A, in the conceived architecture, concentrators get the household level measurements from the smart meters and compute a local SE (note that concentrators receive data with the specific smart meter communication protocol, e.g. DLMS/COSEM, and that the protocol translation into MQTT is only applied for the communication to the cloud platform). The results at the LV feeder bus are then sent through MQTT to the cloud using a specific topic linked to the portion of LV grid to which the concentrator is connected. LV estimators are subscribed to get the data published under that topic and thus they receive the results of the concentrators they subtend (via the MQTT broker). In addition, possible additional meters available in the LV grid also send their measurements, via MQTT, to the cloud, and LV estimators subscribe to receive these measurements.

These inputs are processed to obtain the LV grid SE results, which are then sent to the cloud, via MQTT. Finally, the MV grid SE subscribes to receive the SE results at the

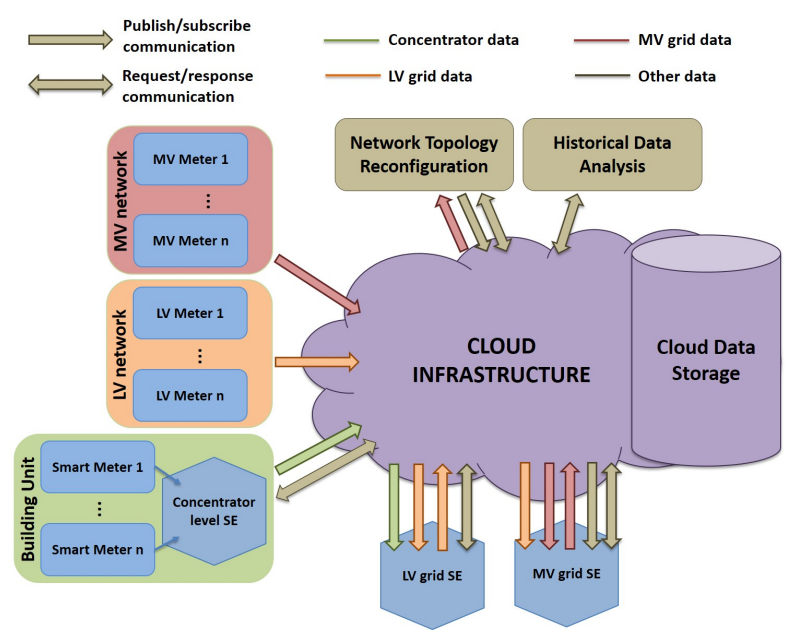

Fig. 4. Communication flow for SEs and auxiliary services

MV/LV substations and the possible measurements coming from additional equipment installed at MV level. The MV estimator can thus perform the MV grid SE and it provides the results to the cloud.

In the proposed platform, two auxiliary services play an important role for the proper execution of the SE processes. First, in a fully automated distribution grid, a network topology reconfiguration algorithm can be used to decide the optimal configuration of the network based on the operating conditions. In this context, a reconfiguration service would subscribe to obtain the results of the MV grid SE and use these data to decide the optimal configuration of the grid. If any change in the topology occurs, the topology reconfiguration service updates the topology information in the cloud through the REST interface. In this way, the MV grid SE (assuming that only the MV grid can be reconfigured) can subscribe to get an alarm whenever a topology change is uploaded in the cloud and then it can request the updated topology. This approach allows requiring and updating the topology information needed for the MV grid SE only when a change occurs.

A second application complementary to the SEs is the historical data analysis service. This service collects the SE results and analyses the historical dataset to provide forecast measurements of power consumption at the different nodes of the grid (depending on day and time of the day). This service provides a back-up for the real-time information in case of lost measurements or bad data identification. Whenever some measurements are missing (or erroneous) in the LV or MV grid $\mathrm{SE}$, these can be thus replaced by retrieving from the cloud a corresponding pseudo-measurement based on the historical data. A request/response communication is used in this case since these data are needed only when a missing or bad measurement is detected. While these services are essential for the estimators (to ensure the use of the correct topology and to guarantee the observability of the grids), a deeper description of these services is out of the scope of this paper (details on the coordinated operation of DSSE and network topology reconfiguration services can be found in [27]). 


\section{DSSE PERFORMANCE ANALYSIS}

In this section, the potential performance achievable through the proposed multi-level SE architecture are analysed in terms of SE accuracy. To this purpose, the following assumptions are considered

- The grid is provided with a full coverage of smart meter at the end-users nodes: this will be a realistic assumption for many countries in the next future [37].

- Smart meters can provide real-time measurements of voltage and power: nowadays, smart meters are mainly deployed for billing purposes; however, some Distribution System Operators (DSOs) are already upgrading their functionalities to enable their use for automation purposes (see [23], [26]).

- Smart meters have time synchronization: this is often achieved distributing a time reference through the network; possible effects of lack of synchronization have been investigated in [38] and are not considered here.

- Topology and network data are available: sometimes LV grid data can be unknown (see for example [19]), but for the application of WLS SE, as in this paper, the knowledge of the network characteristics is essential. Since many DSOs are currently proceeding with the digitalization of the grid data, it is likely that this information will be available for many grids in future.

\section{A. Voltage profile accuracy}

The evaluation of the DSSE performance in term of voltage profile accuracy is performed through the analysis of the covariance matrix of the estimates given by the inverse of the Gain matrix. Let us consider the following state vector $\mathbf{x}$ :

$$
\mathbf{x}=\left[V_{a}, V_{b}, V_{c}, \mathbf{i}_{r e, a}, \mathbf{i}_{i m, a}, \mathbf{i}_{r e, b}, \mathbf{i}_{i m, b}, \mathbf{i}_{r e, c}, \mathbf{i}_{i m, c}\right]
$$

where $V_{\phi}$ with $\phi=\{a, b, c\}$ is the voltage magnitude at the phase $\phi$ of the reference bus, whereas $\mathbf{i}_{r e, \phi}$ and $\mathbf{i}_{i m, \phi}$ are the vectors of the real and the imaginary components of the branch currents for phase $\phi$.

Given this form of the state vector, the covariance matrix of the estimates can be written as:

$$
\boldsymbol{\Sigma}_{\mathrm{x}}=\left[\begin{array}{cc}
\boldsymbol{\Sigma}_{\mathbf{V}} & \boldsymbol{\Sigma}_{\mathbf{V}, \mathbf{i}} \\
\boldsymbol{\Sigma}_{\mathbf{i}, \mathrm{V}} & \boldsymbol{\Sigma}_{\mathbf{i}}
\end{array}\right]=\left[\begin{array}{cc}
\mathbf{G}_{\mathbf{V}} & \mathbf{G}_{\mathbf{V}, \mathbf{i}} \\
\mathbf{G}_{\mathbf{i}, \mathbf{V}} & \mathbf{G}_{\mathbf{i}}
\end{array}\right]^{-1}
$$

where $\boldsymbol{\Sigma}_{\mathbf{V}}$ is the $3 \times 3$ submatrix of covariances of the voltage magnitude estimates, $\boldsymbol{\Sigma}_{\mathbf{i}}$ is the covariance matrix of the branch currents and $\boldsymbol{\Sigma}_{\mathbf{V}, \mathbf{i}}$ and $\boldsymbol{\Sigma}_{\mathbf{i}, \mathbf{V}}$ are the covariances between voltage and current variables. The same subscripts are also used to split in a similar way the Gain matrix.

Focusing on the submatrix $\boldsymbol{\Sigma}_{\mathbf{V}}$, as shown in [39] and [40], using the properties for the inverse of a block matrix and the Woodbury identity relationship, $\boldsymbol{\Sigma}_{\mathbf{V}}$ can be computed as:

$$
\boldsymbol{\Sigma}_{\mathbf{V}}=\mathbf{G}_{V}^{-1}+\mathbf{G}_{V}^{-1} \cdot \mathbf{G}_{V, i} \cdot \boldsymbol{\Sigma}_{\mathbf{i}} \cdot \mathbf{G}_{i, V} \cdot \mathbf{G}_{V}^{-1}
$$

As presented in [39], the first component of uncertainty in (10) is only associated to the number and accuracy of the available voltage measurements, whereas the second term is linked (through the impedances present in $\mathbf{G}_{V, i}$ and $\mathbf{G}_{i, V}$ and the branch current uncertainties in $\boldsymbol{\Sigma}_{\mathbf{i}}$ ) to the voltage drops in the path between each voltage measurement location and the considered reference bus. If voltage drops and branch current uncertainties are low (see in [39] the effect of power injection accuracy on the uncertainty of the voltage profile), their contribution to the overall uncertainty in $\boldsymbol{\Sigma}_{\mathbf{V}}$ is very small and can be in first approximation disregarded. In this way, the voltage uncertainty computation is reduced to the only calculation of the inverse of the Gain submatrix $\mathbf{G}_{V}$.

At concentrator level, voltage measurements are only linked to the voltage state at the same phase of the measurement and no coupling exists with the voltage states of the other phases. The computation of the submatrix $\mathbf{G}_{V}$ thus results in a diagonal matrix having the diagonal elements equal to:

$$
\mathbf{G}_{V}(\phi, \phi)=\sum_{i=1}^{M_{\phi, c o n c}} w_{V_{i, \phi}}
$$

where $w_{V_{i}}$ is the weight of $i$-th voltage measurement for phase $\phi$ and $M_{\phi, c o n c}$ is the total number of smart meters at phase $\phi$ for the considered concentrator. The inverse of the submatrix is simply given by the inverse of the terms in (11). Considering that the WLS weights have to be chosen as the inverse of the measurement variance, if all the smart meters have the same voltage accuracy $U_{V \%}$, from (11), the uncertainty of the voltage estimation in the concentrator $\mathrm{SE}$ can be found to be:

$$
U_{\hat{V}, \phi, \text { conc } \%} \simeq \frac{U_{V \%}}{\sqrt{M_{\phi, \text { conc }}}}
$$

Such a result highlights that the higher the number of smart meter measurements, the better the voltage estimation.

Considerations similar to those made for the concentrator SE apply also to the LV grid SE. The submatrix $\mathbf{G}_{V}$ still results in a diagonal matrix where the diagonal terms are equal to the sum of the weights of the input voltage measurements: however, in this case, the input voltage measurements are the voltage estimation results obtained at the concentrator level. Equation (11) thus becomes:

$$
\mathbf{G}_{V}(\phi, \phi)=\sum_{i=1}^{C_{\phi, L V}} w_{V_{i, \phi}}
$$

where $C_{\phi, L V}$ is the number of concentrators at phase $\phi$ subtended by the LV grid. Given the uncertainty in (12) as input for the calculation of the weights in (13), the following uncertainty can be obtained for the LV grid voltage estimation:

$$
U_{\hat{V}, \phi, L V \%} \simeq \frac{U_{V \%}}{\sqrt{M_{\phi, L V}}}
$$

where $M_{\phi, L V}$ is the total number of smart meters connected to the LV grid (at phase $\phi$ ). Equation (14) clearly shows that the integration of the DSSE hierarchical levels as done in this work leads to a significant improvement of the voltage estimation accuracy when moving from concentrator to LV SE and all the smart meter measurements contribute to the enhancement of the accuracy performance.

While the mathematical framework is the same also for the evaluation of the voltage accuracy at MV level, in this case the 
uncertainty calculation has to take into account the coupling among phases introduced by the delta-star transformer. As visible in (7), the Jacobian of each voltage measurement involves two different phases. Due to the phase shift $\left(30^{\circ}\right)$ brought by the delta-star connection, the derivative with respect to the voltage states of each involved phase is equal to $\frac{\sqrt{3}}{2}$. Considering the contribution of the voltage measurements at all the phases, the submatrix $\mathbf{G}_{V}$ becomes:

$\mathbf{G}_{V}=\frac{3}{4} \cdot\left[\begin{array}{ccc}W_{V, A}+W_{V, B} & W_{V, B} & W_{V, A} \\ W_{V, B} & W_{V, B}+W_{V, C} & W_{V, C} \\ W_{V, A} & W_{V, C} & W_{V, A}+W_{V, C}\end{array}\right]$

where the generic term $W_{V, \phi}$ is:

$$
W_{V, \phi}=\sum_{i=1}^{N_{\phi}} w_{V_{i, \phi}}
$$

with $N_{\phi}$ being the total number of voltage measurements for phase $\phi$. The inverse of the submatrix $\mathbf{G}_{V}$ gives the following result for all the diagonal elements (which correspond to the variances of the voltage states):

$$
\boldsymbol{\Sigma}_{V}(\phi, \phi)=\frac{W_{V, A} W_{V, B}+W_{V, B} W_{V, C}+W_{V, A} W_{V, C}}{3 W_{V, A} W_{V, B} W_{V, C}}
$$

Taking into account that the voltage measurement weights are given by the uncertainties obtained during the LV SE processes (eq. (14)), from (17) it is possible to obtain the following theoretical uncertainty:

$$
U_{\hat{V}, \phi, M V \%} \simeq U_{V \%} \cdot \sqrt{\frac{M_{A} M_{B}+M_{B} M_{C}+M_{A} M_{C}}{M_{A} M_{B} M_{C}}}
$$

where $M_{\phi}$ is the overall number of smart meter measurements subtended by the MV grid for phase $\phi$.

If the number of smart meters for the different phases is almost the same (namely $M_{A} \simeq M_{B} \simeq M_{C}=M$ ), equation (18) can be further simplified and it becomes:

$$
U_{\hat{V}, \phi, M V \%} \simeq \frac{\sqrt{3} U_{V \%}}{\sqrt{M}}
$$

From (19) it is possible to observe how, in the proposed multilevel framework, the whole number of smart meters subtended by the MV grid contributes to decrease the uncertainty of the MV voltage estimation profile.

\section{B. Power flow accuracy}

The analysis of the DSSE performance in terms of power flow accuracy can be performed through a backward sweep procedure, starting from the leaf nodes of the grid and moving towards the root bus. Power losses in the grid are neglected in first approximation to simplify the analysis.

At concentrator level, the total power injection seen from the LV feeder bus is equal to the sum of the power consumed (or generated) by each end-user. Given a relative uncertainty $U_{P \%}$ for the power measurements of the smart meters (same considerations apply for both active and reactive power), the total uncertainty for the power injection at the feeder bus is:

$$
U_{\hat{P}, \phi, \text { conc } \%} \simeq \frac{U_{P \%} \cdot \sqrt{\sum_{i=1}^{M_{\phi, c o n c}} P_{i}^{2}}}{\sum_{i=1}^{M_{\phi, c o n c}} P_{i}}
$$

Equation (20) shows that the power uncertainty strongly depends on the values of consumption and generation of the customers. A simplified case occurs when all the customers are consuming (or generating) power. In this case, the power injection uncertainty falls within the following boundaries:

$$
\frac{U_{P \%}}{\sqrt{M_{\phi, \text { conc }}}} \leq U_{\hat{P}, \phi, \text { conc } \%} \leq U_{P \%}
$$

The upper boundary in (21) occurs when the total power injection at the feeder bus is only given by a single customer, whereas the lower boundary occurs when the total power injection is equally divided among all the downstream users. In general, the more uniform the distribution of the power among the customers, the lower the final uncertainty. In this particular case, (21) shows that the estimation uncertainty for the power injection at the LV feeder bus will be always lower than the starting measurement uncertainty of the smart meters.

For the LV grid SE, similar considerations also apply. The power flow uncertainty in each branch of the grid is given by the contribution of all the customer injections downstream that branch. At the MV/LV substation, thus, the following holds:

$$
U_{\hat{P}, \phi, L V \%} \simeq \frac{U_{P \%} \cdot \sqrt{\sum_{i=1}^{M_{\phi, L V}} P_{i}^{2}}}{\sum_{i=1}^{M_{\phi, L V}} P_{i}}
$$

For the particular case of customers that are all consuming (or generating) power, the boundaries in (21) still hold, but the lower boundary will take into account the total amount of customers subtended to the LV grid:

$$
\frac{U_{P \%}}{\sqrt{M_{\phi, L V}}} \leq U_{\hat{P}, \phi, L V \%} \leq U_{P \%}
$$

At the MV level, the propagation of the uncertainties is affected by the presence of the delta-star transformer. Referring to the same scheme depicted in Fig. 2, the active and reactive power injections seen from the MV side (node $m$ ) can be written as (considering phase $a$ as example):

$$
\begin{aligned}
P_{m, a}^{i n j} & =V_{m, a} \frac{P_{n, a}^{i n j} \cos \left(\theta_{m_{a} n_{a}}\right)-Q_{n, a}^{i n j} \sin \left(\theta_{m_{a} n_{a}}\right)}{V_{n, a}}+ \\
& -V_{m, a} \frac{P_{n, b}^{i n j} \cos \left(\theta_{m_{a} n_{b}}\right)-Q_{n, b}^{i n j} \sin \left(\theta_{m_{a} n_{b}}\right)}{V_{n, b}} \\
Q_{m, a}^{i n j} & =V_{m, a} \frac{Q_{n, a}^{i n j} \cos \left(\theta_{m_{a} n_{a}}\right)+P_{n, a}^{i n j} \sin \left(\theta_{m_{a} n_{a}}\right)}{V_{n, a}}+ \\
& -V_{m, a} \frac{Q_{n, b}^{i n j} \cos \left(\theta_{m_{a} n_{b}}\right)+P_{n, b}^{i n j} \sin \left(\theta_{m_{a} n_{b}}\right)}{V_{n, b}}
\end{aligned}
$$

where $P_{n, \phi}^{i n j}$ and $Q_{n, \phi}^{i n j}$ are the active and reactive power injections seen from the phase $\phi$ of node $n, V_{n, \phi}$ is the voltage 
magnitude at phase $\phi$ of node $n$, and $\theta_{m_{\phi_{1}} n_{\phi_{2}}}$ is the phase angle difference between the voltages at phase $\phi_{1}$ of node $m$ and the current at phase $\phi_{2}$ of node $n$.

Considering, in first approximation, the voltages equal to their nominal value and taking into account the phase shift brought by the delta-star connection, the following simplified relationships can be obtained:

$$
\begin{gathered}
P_{m, a}^{i n j} \simeq \frac{P_{n, a}^{i n j}+P_{n, b}^{i n j}}{2}-\frac{Q_{n, a}^{i n j}-Q_{n, b}^{i n j}}{2 \sqrt{3}} \\
Q_{m, a}^{i n j} \simeq \frac{Q_{n, a}^{i n j}+Q_{n, b}^{i n j}}{2}+\frac{P_{n, a}^{i n j}-P_{n, b}^{i n j}}{2 \sqrt{3}}
\end{gathered}
$$

From (26) and (27), it is possible to derive the resulting uncertainty for the MV power injections:

$$
\begin{aligned}
& U_{\hat{P}_{m, a, M V}^{i n j}} \simeq \sqrt{\frac{U_{P_{n, a}^{i n j}}^{2}+U_{P_{n, b}^{i n j}}^{2}}{4}+\frac{U_{Q_{n, a}^{i n j}}^{2}+U_{Q_{n, b}^{i n j}}^{2}}{12}} \\
& U_{\hat{Q}_{m, a, M V}^{i n j}} \simeq \sqrt{\frac{U_{Q_{n, a}^{i n j}}^{2}+U_{Q_{n, b}^{i n j}}^{2}}{4}+\frac{U_{P_{n, a}^{i n j}}^{2}+U_{P_{n, b}^{i n j}}^{2}}{12}}
\end{aligned}
$$

where $U_{P_{n, a}^{i n j}}$ and $U_{Q_{n, a}^{i n j}}$ are the uncertainties (in absolute value) for the power injections resulting at the MV side of the transformer due to the LV grid estimations.

Starting from the power injection uncertainties in (28) and (29), the uncertainty for the power flows in each branch of the MV grid is then given by the quadratic sum of the power injection uncertainties at all the nodes downstream the branch. At the primary substation, the following holds (a similar equation can be also derived for the reactive power):

$$
U_{\hat{P}, \phi, M V \%} \simeq \frac{\sqrt{\sum_{i=1}^{N_{M V}} U_{P_{i, \phi, M V}^{i n j}}^{2}}}{\sum_{i=1}^{N_{M V}} P_{i, \phi}^{i n j}}
$$

where $N_{M V}$ is the total number of MV buses.

The above relationships show how the uncertainties propagate from the LV grid estimators to the MV grid level. It is important to note that these relationships are a rough calculation since the voltages in (24) and (25) have been approximated (and their uncertainties neglected) and calculations only took into account the contribution to uncertainty provided by the power injections. However, when accurate voltage measurements are available at the different nodes (as it is at the MV level according to the analytical findings in Section IV-A), they also contribute to the power flow estimation because they put a constraint on the voltage drops between buses. As a result, depending on the scenario, slight over- or underestimations of the uncertainty can be found. Nevertheless, these relationships can be used as a good benchmark by DSOs to evaluate if the achievable accuracies are in line with their expectations or requirements for the grid monitoring.

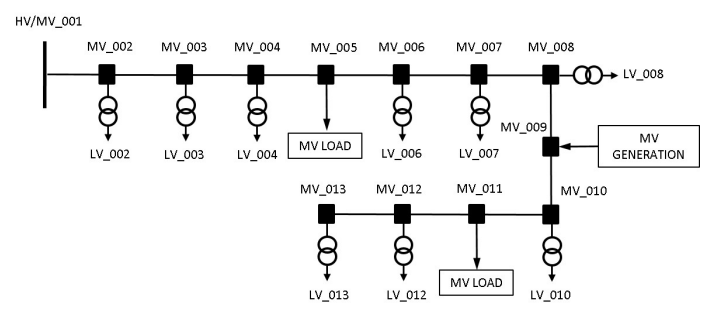

Fig. 5. MV feeder used for the simulations.

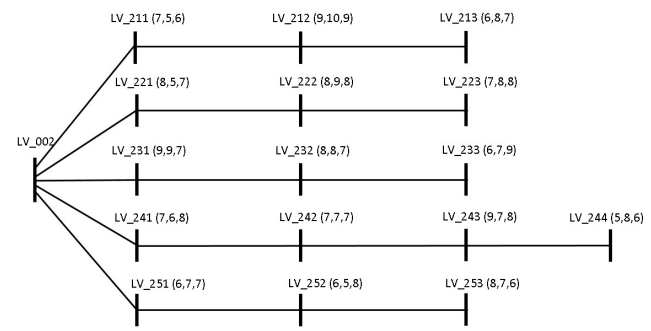

Fig. 6. Example of LV grid topology used for the simulations.

\section{Tests And Results}

The scenario used for the simulations is composed of a 13bus MV feeder connected to $9 \mathrm{MV} / \mathrm{LV}$ substations, $2 \mathrm{MV}$ loads and a PV plant (see Fig. 5). For each MV/LV substation, the downstream LV grid has been modelled down to the building units. Fig. 6 shows, as an example, one of the LV grids with the indication of the LV feeder buses and of the subtended customers for each of the three phases. Daily profiles have been generated for each LV residential customer and for the MV loads and generation. Smart meters have been assumed to be available at all the load and generation nodes with accuracies equal to $1 \%$ and $2 \%$ for the voltage and power measurements, respectively. Different simulations have been performed to show the performance of the proposed multilevel architecture. The performed tests mainly aim at proving the validity of the accuracy analysis performed in Section IV and to highlight the benefits given by the conceived multi-level architecture.

\section{A. Validation of the accuracy performance analysis}

In this subsection, off-line Monte Carlo (MC) simulations have been first performed to evaluate the accuracy performance of the designed multi-level SE and to validate the analytical relationships shown in Section IV. Simulations have been then run also in real-time to show the operation of the DSSE in coordination with the cloud platform.

Off-line MC simulations have been carried out by considering a specific time step of the day. For such time step, a power flow calculation is performed to obtain the reference operating conditions of the grid. Measurements are then extracted by adding random noise to the reference electrical quantities according to a Gaussian probability distribution with standard deviation equal to one third of the related accuracy value. For each MC simulation, 25000 trials have been run by extracting different measurements in order to have a statistically meaningful dataset. 
TABLE I. CONCENTRATOR SE ACCURACY RESULTS

\begin{tabular}{c|c|c|c|c|c}
\hline \multirow{2}{*}{ Node } & \multicolumn{2}{|c|}{ Voltage Uncertainty [\%] } & \multicolumn{3}{c}{ Power Uncertainty [\%] } \\
\cline { 2 - 6 } & Theor. & MC & Theor. LB & Theor. UB & MC \\
\hline LV 211 ph.A & 0.38 & 0.38 & 0.76 & 2.00 & 1.23 \\
LV 211 ph.B & 0.45 & 0.45 & 0.89 & 2.00 & 1.64 \\
LV 211 ph.C & 0.41 & 0.41 & 0.82 & 2.00 & 0.99 \\
\hline
\end{tabular}
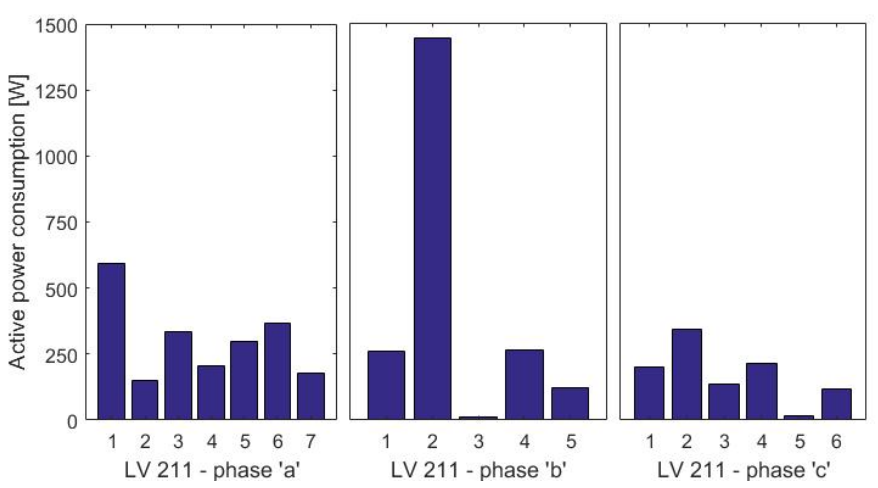

Fig. 7. Power consumption of single customers subtended by concentrator at bus LV_211

Table I shows, as an example, the results obtained for the concentrator located in bus LV_211, which has 7, 5 and 6 customers connected to phase $a, b$ and $c$, respectively. The Table reports the theoretical values of uncertainty given by the mathematical relationships found in Section IV and the experimental results obtained through the MC simulation. For the power injection, since all the residential customers are passive users, the lower and upper bounds (LB and UB in the Table, respectively) of uncertainty have been indicated. It is possible to note that theoretical and experimental voltage uncertainties perfectly match and power injection uncertainties are within the expected boundaries. Power uncertainties can be more or less close to the lower bound depending on the distribution of the power among the subtended customers. Fig. 7 shows the values of power consumption, at the considered time step, for the customers connected to the different phases. It is possible to observe that phase $c$ has a quite uniform distribution of the power consumptions and this is reflected in the uncertainty result, which is quite close to the lower bound. On the other hand, in phase $b$, the contribution to the total uncertainty is mainly given by one of the customers and hence the final power injection uncertainty is only slightly better than the starting smart meter uncertainty.

At the LV grid level, a much larger number of smart meter measurements allows refining the SE results. Table II shows

TABLE II. LV GRID SE ACCURACY RESULTS

\begin{tabular}{c|c|c|c|c|c}
\hline \multirow{2}{*}{ Node } & \multicolumn{2}{|c|}{ Voltage Uncertainty [\%] } & \multicolumn{3}{c}{ Power Uncertainty [\%] } \\
\cline { 2 - 6 } & Theor. & MC & Theor. LB & Theor. UB & MC \\
\hline LV 002 ph.A & 0.09 & 0.09 & 0.19 & 2.00 & 0.31 \\
LV 002 ph.B & 0.09 & 0.09 & 0.19 & 2.00 & 0.37 \\
LV 002 ph.C & 0.09 & 0.09 & 0.18 & 2.00 & 0.35 \\
\hline
\end{tabular}

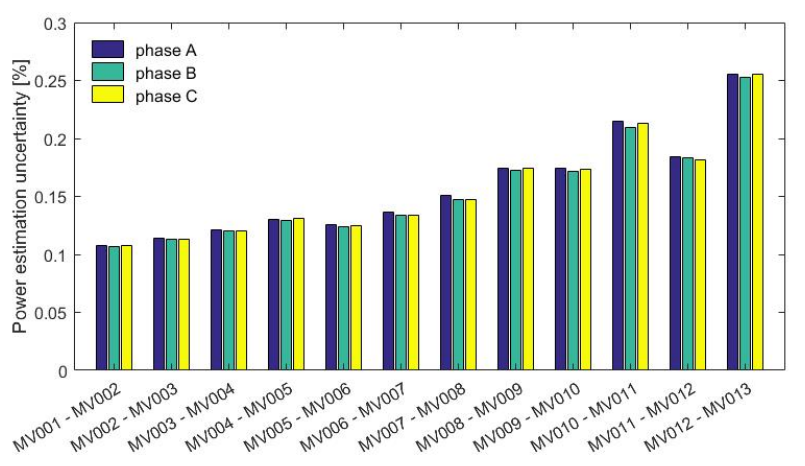

Fig. 8. Profile of power uncertainties at the branches of the MV network

the uncertainty results for the LV grid depicted in Fig. 6. As expected, voltage estimation uncertainty is significantly decreased and a very accurate voltage profile of the grid can be achieved. Similarly, power injection uncertainties are also drastically improved as the total power at the substation is due to the contribution of many end-users. Such results highlight the large benefits potentially achievable by integrating smart meter measurements in a properly designed SE framework.

In the MV grid, the estimation accuracy is further refined thanks to the contribution of all the LV grid SEs. For the voltage profile, an estimation accuracy equal to $0.05 \%$ is obtained through the MC simulations, which is compliant with the analytical relationship in (19) (note that around one thousand residential customers are connected in total to each phase). For the power injections at each MV/LV substation, an accuracy of $0.25 \%$ is achieved, which is in line with the theoretical result that can be found starting from eq. (28). At the branches, the power estimation uncertainty can be obtained as quadratic combination of the power injection uncertainties at the downstream nodes. Fig. 8 shows, as an example, the uncertainties obtained in a time step where no generation is available. It can be observed that very accurate estimations are achieved (better than the starting uncertainty of the smart meters), and these improve when moving towards the root node (as an effect of the quadratic combination of the uncertainties). When generation is present, this trend is not always clearly observable, since the relative terms of uncertainties increase significantly when the branch power tends towards zero. In general, however, high values of relative uncertainty are present only when the branch power is extremely low, whereas low values of uncertainty occur when the branches are heavily loaded (which can be the most critical situation to be monitored). These results prove that smart meter measurements at LV level could be essential to have an accurate monitoring also of the upper-level MV grid, without the need to use inaccurate pseudo-measurements or to have an extensive deployment of MV meters.

As final results, Fig. 9 and 10 show the voltage and power estimations in the MV grid over the day when the designed multi-level estimator is used in a real-time simulation environment with the described cloud platform. In this test, a simulation is carried out for the whole day by using different 


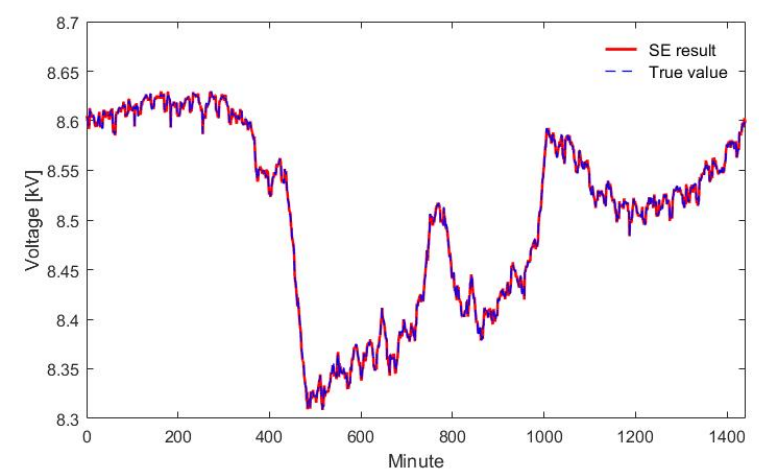

Fig. 9. Voltage estimation profile at phase $a$ of bus MV_013, maximum error: $0.06 \%$

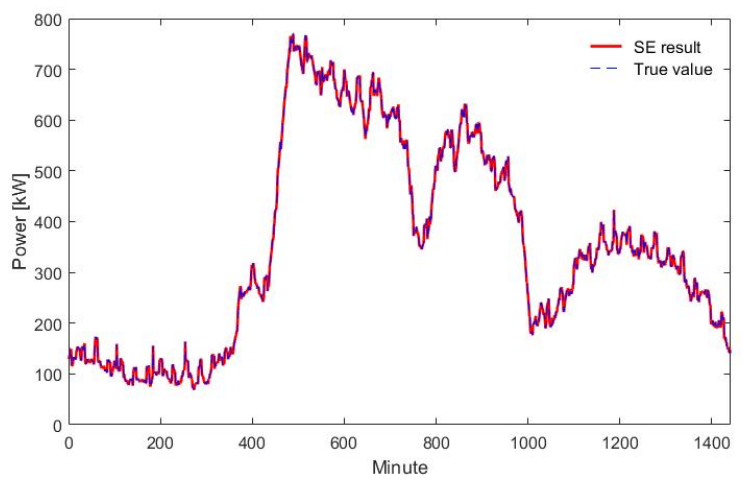

Fig. 10. Active power estimation profile at phase $a$ of the branch MV_001 - MV_002, maximum error: $0.01 \%$

daily profiles for all the loads and generators. To emulate the smart meter measurements, random noise is added to the reference electrical quantities obtained through the power flow calculation. Smart meter measurements are extracted from the power system simulator every minute and used to trigger the SE process. First, the smart meter measurements are processed to perform the concentrator level SE. Then, concentrator level results are sent, through the communication infrastructure decribed in Section III, to the cloud platform for triggering the execution of the estimation steps at LV and MV level.

Fig. 9 shows the estimation of the voltage profile during the whole day for bus MV_013 (phase $a$ ), which is the furthest one from the HV/MV substation and, therefore, the one where the most important voltage drops occur. It is possible to observe that the proposed multi-level SE is able to properly track the voltage variations over time and only very small errors exist (maximum error found was $0.06 \%$ ). Similarly, Fig. 10 depicts the result of power estimation for the branch between nodes MV_001 and MV_002 (phase $a$ ), which is the most loaded one in the network. Even in this case, a very accurate tracking of the flowing power is achieved (maximum error is $0.01 \%$ ). Such results further highlight the potential benefits offered by the designed smart meter based SE framework.
TABLE III. DISTRIBUTED VS CENTRALIZED ARCHITECTURE: TIME PERFORMANCE

\begin{tabular}{c|c|c|c|c}
\hline & \multicolumn{3}{|c|}{ Distributed architecture } & \multirow{2}{*}{$\begin{array}{c}\text { Centralized } \\
\text { architecture }\end{array}$} \\
\cline { 2 - 4 } & Conc. level & LV level & MV level & $11.6 \mathrm{~s}$ \\
Min time & $1.5 \mathrm{~ms}$ & $3.6 \mathrm{~ms}$ & $7.0 \mathrm{~ms}$ & $11.9 \mathrm{~s}$ \\
Avg time & $2.0 \mathrm{~ms}$ & $4.7 \mathrm{~ms}$ & $7.5 \mathrm{~ms}$ & $13.4 \mathrm{~s}$ \\
Max time & $3.7 \mathrm{~ms}$ & $7.5 \mathrm{~ms}$ & $23.9 \mathrm{~ms}$ & \\
\hline
\end{tabular}

\section{B. Benefits of the proposed multi-level framework}

In the proposed multi-level framework, two aspects play a major role for dealing with the challenges associated to the deployment of DSSE: i) the distributed nature of the architecture, which allows distributing the computational requirements of SE and shortening the computation time of the SE algorithms; ii) the use of the WLS formulation, which allows properly coordinating the different SE levels to maximize the achievable accuracy performance. In this subsection, simulations are presented to show these features.

In the first test, the distributed architecture is compared to a centralized one. To this purpose, the performances of the SE algorithms used at the different SE levels are compared to those of a centralized estimator dealing with both the LV and the MV grid. Table III shows the results obtained over 1000 MC iterations. Tests were performed using Matlab 2016 in a PC having a $3.3 \mathrm{GHz}$ CPU and $8 \mathrm{~GB}$ of RAM. The main advantage of the proposed architecture is the possibility to parallelize the execution of all the algorithms running at the same hierarchical level (all the concentrators SEs at the concentrator level and the LV SEs at the LV grid level run in parallel). Moreover, each estimator has only to deal with a small portion of the grid (the maximum number of nodes is in the MV grid model, which has 21 three-phase buses). As a result, the algorithms implemented in the distributed architecture take a very short time to perform their estimation (in the order of few $\mathrm{ms}$ ). On the contrary, the centralized algorithm takes several seconds (up to $13.4 \mathrm{~s}$ in the performed test) to handle the complete grid, which has 3498 buses (note that sparse matrices have been used, where convenient, to shorten the execution times). In [27], more details on the time performance of the distributed architecture are provided for the time needed by the MQTT interfaces to publish or receive the data and for the MQTT communication (in the order of tens and hundreds of $\mathrm{ms}$, respectively). Considering the sum of all the contribution in the distributed architecture, the overall execution times are generally below $1 \mathrm{~s}$. This proves that the proposed architecture clearly outperforms a centralized solution in terms of time performance and this trend would be even more evident in case of larger distribution grids. Such an improvement in the computation performance is however obtained at the expenses of a slight degradation of the estimation accuracy. This holds above all for the LV grids, where voltage estimation uncertainties around $0.04 \%$ can be achieved at both the LV feeder and the end-user buses when adopting a centralized estimator. As shown in Tables I and II, with the proposed architecture, the voltage uncertainties are instead around $0.40 \%$ and $0.09 \%$ at the concentrator and LV level, respectively. 
TABLE IV. PERFORMANCE FOR DIFFERENT SE FORMULATIONS

\begin{tabular}{|c|c|c|c|c|c|c|c|c|c|}
\hline \multirow{2}{*}{ Index } & \multicolumn{3}{|c|}{ WLS } & \multicolumn{3}{|c|}{$\mathrm{B} / \mathrm{F}$} & \multicolumn{3}{|c|}{ LAV } \\
\hline & Conc & LV & MV & Conc & LV & MV & Conc & LV & MV that may be achieved, in terms of reduction of the computation \\
\hline $\begin{array}{l}\text { Average } \\
\text { time }[\mathrm{ms}]\end{array}$ & 2.0 & 4.7 & 7.5 & 0.2 & 0.5 & 2.7 & 155 & 235 & 139 time, thanks to the proposed multi-level framework. To give \\
\hline $\begin{array}{l}\text { Average } \\
\text { iteration no. }\end{array}$ & 3.0 & 4.5 & 5.0 & 3.0 & 4.7 & 4.0 & 3.2 & 4.6 & $\begin{array}{l}\text { 5.4 complete overview of the conceived multi-level DSSE a } \\
\text { chitecture, the paper also presents a cloud-based infrastructur }\end{array}$ \\
\hline $\begin{array}{l}\text { Voltage } \\
\text { unc. [\%] }\end{array}$ & 0.37 & 0.09 & 0.05 & 1.00 & 1.01 & 1.04 & 0.47 & 0.15 & 0.10 used to allow the interconnection of the different hierarchical \\
\hline $\begin{array}{l}\text { Power } \\
\text { unc. }[\%]\end{array}$ & 2.00 & 0.85 & 1.11 & 2.00 & 0.85 & 1.36 & 2.00 & 0.85 & $\begin{array}{l}1.15 \text { levels. The same infrastructure can also be used to coordinate } \\
\text { DSSE with other functions for distribution grid management. }\end{array}$ \\
\hline
\end{tabular}

A second test is run to show the benefits given by the WLS formulation and the associated coordination framework. While some literature already shows the advantages of WLS over other formulations (see for example [41]), the goal here is to highlight the main implications in terms of accuracy and time execution for the presented architecture. To this purpose, the performance of the proposed WLS-based framework are compared to those obtained by integrating different SE formulations in the multi-level architecture. The Backward/Forward (B/F) sweep technique is used as main term of comparison, since it is an alternative approach widely used in distribution radial grids [42], [43]. In addition, the Least Absolute Value (LAV) approach is also tested, since it is an approach often used in the SE context [29].

Table IV gives the results obtained over $1000 \mathrm{MC}$ trials with the details for each hierarchical level. In particular, the average execution time and number of iterations are provided to evaluate the computational performance, while the estimation uncertainty of node voltages and branch active powers is given to assess the accuracy behaviour (the given values are the average among the expanded uncertainties obtained in all the nodes or branches of the considered hierarchical level). In Table IV, it is possible to observe that the $\mathrm{B} / \mathrm{F}$ algorithm have the fastest convergence. However, it also exhibits an important degradation of the voltage estimation accuracy. This is because this approach does not allow the integration of all the available voltage measurements, but only uses one of them for the computation of the backward sweep (see [42]). The LAV approach allows instead having accuracy performance closer to those of the WLS framework, but the needed optimization is computational demanding and this leads to an evident increase of the execution times. Such results thus highlight that the proposed WLS framework is a valid solution to maximize the accuracy performance, exploiting all the smart meter measurements, while guaranteeing sufficiently low execution times.

\section{CONCLUSIONS}

This paper presented the design of a multi-level SE aimed at monitoring both LV and MV grids through the use of smart meter measurements. A mathematical analysis has been developed to derive the analytical expressions needed to compute the uncertainties of voltages and powers at all the levels of the $\mathrm{SE}$ architecture. Such findings have been validated by means of dedicated simulations and show how the integration of a large number of smart meters is beneficially reflected into the

\section{ACKNOWLEDGEMENTS}

This work was supported by Flexmeter and Sogno, which are EU Horizon 2020 projects under grant agreement no. 646568 and 774613 , respectively.

\section{REFERENCES}

[1] J. Fan and S. Borlase, "The evolution of distribution," Power and Energy Magazine, IEEE, vol. 7, no. 2, pp. 63-68, March 2009.

[2] G. Heydt, "The next generation of power distribution systems," IEEE Trans. Smart Grid, vol. 1, no. 3, pp. 225-235, Dec. 2010.

[3] A. Meliopoulos, E. Polymeneas, Z. Tan, R. Huang, and D. Zhao, "Advanced distribution management system," IEEE Trans. Smart Grid, vol. 4, no. 4, pp. 2109-2117, Dec 2013.

[4] M. Baran and A. Kelley, "A branch-current-based state estimation method for distribution systems," IEEE Trans. Power Syst., vol. 10, no. 1, pp. 483-491, Feb. 1995.

[5] W. M. Lin and J. H. Teng, "Distribution fast decoupled state estimation by measurement pairing," IET Gener. Transm. Distrib., vol. 143, no. 1, pp. 43-48, 1996.

[6] H. Wang and N. Schulz, "A revised branch current-based distribution system state estimation algorithm and meter placement impact," IEEE Trans. Power Syst., vol. 19, no. 1, pp. 207-213, Feb. 2004.

[7] D. A. Haughton and G. T. Heydt, "A linear state estimation formulation for smart distribution systems," IEEE Trans. Power Syst., vol. 28, no. 2, pp. 1187-1195, May 2013.

[8] M. Pau, P. A. Pegoraro, and S. Sulis, "Efficient branch-current-based distribution system state estimation including synchronized measurements," IEEE Trans. Instrum. Meas., vol. 62, no. 9, pp. 2419-2429, Sep. 2013.

[9] D. Della Giustina, M. Pau, P. A. Pegoraro, F. Ponci, and S. Sulis, "Electrical distribution system state estimation: measurement issues and challenges," IEEE Instrumentation Measurement Magazine, vol. 17, no. 6, pp. 36-42, Dec. 2014.

[10] A. Bernieri, G. Betta, C. Liguori, and A. Losi, "Neural networks and pseudo-measurements for real-time monitoring of distribution systems," IEEE Trans. Instrum. Meas., vol. 45, no. 2, pp. 645-650, Apr 1996.

[11] E. Manitas, R. Singh, B. Pal, and G. Strbac, "Distribution system state estimation using an artificial neural network approach for pseudo measurement modeling," IEEE Trans. Power Syst., vol. 27, no. 4, pp. 1888-1896, Nov. 2012.

[12] A. Angioni, T. Schlösser, F. Ponci, and A. Monti, "Impact of pseudomeasurements from new power profiles on state estimation in lowvoltage grids," IEEE Trans. Instrum. Meas., vol. 65, no. 1, pp. 70-77, Jan 2016.

[13] C. Muscas, F. Pilo, G. Pisano, and S. Sulis, "Optimal placement of measurement devices in electric distribution systems," in 2006 IEEE Instrumentation and Measurement Technology Conference Proceedings, April 2006, pp. 1873-1878.

[14] R. Singh, B. C. Pal, and R. B. Vinter, "Measurement placement in distribution system state estimation," IEEE Trans. Power Syst., vol. 24, no. 2, pp. 668-675, May 2009. 
[15] J. Liu, F. Ponci, A. Monti, C. Muscas, P. A. Pegoraro, and S. Sulis, "Optimal meter placement for robust measurement systems in active distribution grids," IEEE Trans. Instrum. Meas., vol. 63, no. 5, pp. 10961105, May 2014.

[16] M. Ghasemi Damavandi, V. Krishnamurthy, and J. Marti, "Robust meter placement for state estimation in active distribution systems," IEEE Trans. Smart Grid, vol. 6, no. 4, pp. 1972-1982, July 2015.

[17] S. Bhela, V. Kekatos, and S. Veeramachaneni, "Enhancing observability in distribution grids using smart meter data," IEEE Transactions on Smart Grid, vol. 9, no. 6, pp. 5953-5961, Nov 2018.

[18] M. M. Othman, M. H. Ahmed, and M. M. A. Salama, "A novel real time estimation technique for active unbalanced distribution networks using smart meters," in 2018 IEEE Canadian Conference on Electrical Computer Engineering (CCECE), May 2018, pp. 1-5.

[19] F. Ni, P. H. Nguyen, J. F. G. Cobben, H. E. van den Brom, and D. Zhao, "Uncertainty analysis of aggregated smart meter data for state estimation," in 2016 IEEE International Workshop on Applied Measurements for Power Systems (AMPS), Sept 2016, pp. 1-6.

[20] Q. Chen, D. Kaleshi, Z. Fan, and S. Armour, "Impact of smart metering data aggregation on distribution system state estimation," IEEE Trans. Ind. Informat., vol. 12, no. 4, pp. 1426-1437, Aug 2016.

[21] D. Waeresch, R. Brandalik, W. H. Wellssow, J. Jordan, R. Bischler, and N. Schneider, "Linear state estimation in low voltage grids based on smart meter data," in PowerTech, 2015 IEEE Eindhoven, June 2015, pp. 1-6.

[22] A. Abdel-Majeed and M. Braun, "Low voltage system state estimation using smart meters," in 2012 47th International Universities Power Engineering Conference (UPEC), Sept 2012, pp. 1-6.

[23] A. Barbato, A. Dede, D. D. Giustina, G. Massa, A. Angioni, G. Lipari, F. Ponci, and S. Repo, "Lessons learnt from real-time monitoring of the low voltage distribution network," Sustainable Energy, Grids and Networks, 2017. [Online]. Available: http: //www.sciencedirect.com/science/article/pii/S2352467716302089

[24] D. Waeresch, R. Brandalik, W. H. Wellssow, J. Jordan, R. Bischler, and N. Schneider, "Field test of a linear three-phase low-voltage state estimation system based on smart meter data," CIRED - Open Access Proceedings Journal, vol. 2017, no. 1, pp. 1773-1776, 2017.

[25] M. Pau, E. Patti, L. Barbierato, A. Estebsari, E. Pons, F. Ponci, and A. Monti, "Low voltage system state estimation based on smart metering infrastructure," in 2016 IEEE International Workshop on Applied Measurements for Power Systems (AMPS), Sept 2016, pp. 1-6.

[26] A. Angioni, A. Kulmala, D. D. Giustina, M. Mirz, A. Mutanen, A. Ded, F. Ponci, L. Shengye, G. Massa, S. Repo, and A. Monti, "Design and implementation of a substation automation unit," IEEE Transactions on Power Delivery, vol. 32, no. 2, pp. 1133-1142, April 2017.

[27] M. Pau, E. Patti, L. Barbierato, A. Estebsari, E. Pons, F. Ponci, and A. Monti, "A cloud-based smart metering infrastructure for distribution grid services and automation," Sustainable Energy, Grids and Networks, 2017. [Online]. Available: http://www.sciencedirect. com/science/article/pii/S2352467716301783

[28] A. Gomez-Exposito, A. Abur, A. de la Villa Jaen, and C. Gomez-Quiles, "A multilevel state estimation paradigm for smart grids," Proceedings of the IEEE, vol. 99, no. 6, pp. 952-976, June 2011.

[29] A. Abur and A. G. Expòsito, Power System State Estimation. Theory and Implementation. Marcel Dekker, New York, 2004.

[30] M. Pau, P. A. Pegoraro, and S. Sulis, "Performance of three-phase WLS distribution system state estimation approaches," in Applied Measurements for Power Systems (AMPS), 2015 IEEE International Workshop on, Sept 2015, pp. 138-143.

[31] S. Misra, P. V. Krishna, V. Saritha, and M. S. Obaidat, "Learning automata as a utility for power management in smart grids," IEEE Communications Magazine, vol. 51, no. 1, pp. 98-104, 2013.

[32] P. Vytelingum, T. D. Voice, S. D. Ramchurn, A. Rogers, and N. R. Jennings, "Agent-based micro-storage management for the smart grid," in Proceedings of the 9th International Conference on Autonomous Agents and Multiagent Systems: Volume 1, 2010, pp. 39-46.

[33] S. Bera, S. Misra, and J. J. Rodrigues, "Cloud computing applications for smart grid: A survey," IEEE Transactions on Parallel \& Distributed Systems, no. 5, pp. 1477-1494, 2015.

[34] E. Patti, A. L. A. Syrri, M. Jahn, P. Mancarella, A. Acquaviva, and E. Macii, "Distributed software infrastructure for general purpose services in smart grid," IEEE Trans. Smart Grid, vol. 7, no. 2, pp. 1156-1163, March 2016.

[35] E. Patti, A. Acquaviva, and E. Macii, "Enable sensor networks interoperability in smart public spaces through a service oriented approach," in Advances in Sensors and Interfaces (IWASI), 2013 5th IEEE International Workshop on, June 2013, pp. 2-7.

[36] "Message Queue Telemetry Transport (MQTT)," Available: http://mqtt. org/.

[37] The European Commission, "Benchmarking smart metering deployment in the EU-27 with a focus on electricity," Jul. 2014. [Online]. Available: http://eur-lex.europa.eu/legal-content/EN/ TXT/?qid=1403084595595\&uri=COM:2014:356:FIN

[38] A. Alimardani, F. Therrien, D. Atanackovic, J. Jatskevich, and E. Vaahedi, "Distribution system state estimation based on nonsynchronized smart meters," IEEE Trans. Smart Grid, vol. 6, no. 6, pp. 2919-2928, Nov. 2015

[39] M. Pau, P. A. Pegoraro, S. Sulis, and C. Muscas, "Uncertainty sources affecting voltage profile in distribution system state estimation," in Instrumentation and Measurement Technology Conference (I2MTC), 2015 IEEE International, May 2015.

[40] C. Muscas, M. Pau, P. A. Pegoraro, and S. Sulis, "Uncertainty of voltage profile in pmu-based distribution system state estimation," IEEE Trans. Instrum. Meas., vol. 65, no. 5, pp. 988-998, May 2016.

[41] R. Singh, B. Pal, and R. Jabr, "Choice of estimator for distribution system state estimation," IET Gener. Transm. Distrib., vol. 3, no. 7, pp. 666-678, Jul. 2009.

[42] A. Cataliotti, V. Cosentino, D. D. Cara, P. Russotto, E. Telaretti, and G. Tinè, "An innovative measurement approach for load flow analysis in mv smart grids," IEEE Trans. Smart Grid, vol. 7, no. 2, pp. 889-896, March 2016.

[43] A. Cataliotti, C. Cervellera, V. Cosentino, D. D. Cara, M. Gaggero, D. Macciò, G. Marsala, A. Ragusa, and G. Tinè, "An improved load flow method for mv networks based on lv load measurements and estimations," IEEE Trans. Instrum. Meas., pp. 1-18, 2018.

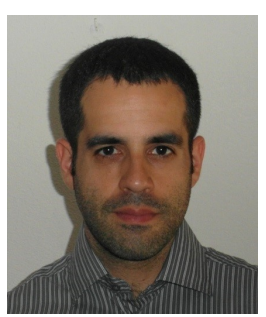

Marco Pau (S'14-M'15) received the M.S. degree (cum laude) in electrical engineering and the Ph.D. degree in electronic engineering and computer science from the University of Cagliari, Cagliari, Italy, in 2011 and 2015, respectively.

$\mathrm{He}$ is currently a Research Associate with the Institute for Automation of Complex Power Systems, E.ON Energy Research Center, RWTH Aachen University, Aachen, Germany, where he is leading the team for Power System Automation. His current research interest include the design of measurement systems for the monitoring and management of active distribution systems and the development of solutions for distribution grid automation. 


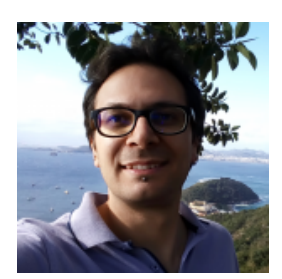

Edoardo Patti (M'17) received his M.Sc. and Ph.D. degrees in Computer Engineering from Politecnico di Torino in 2010 and 2014, respectively.

Currently, he is Assistant Professor at Politecnico di Torino. His research interests concern: ubiquitous computing, Internet of Things, smart systems and cities, software architectures with particular emphasis on infrastructure for Ambient Intelligence, software solutions for simulating and optimising energy systems and software solutions for energy data visualisation to increase user awareness. In the fields above, he has authored over 70 scientific publications between 2011 and 2018 .

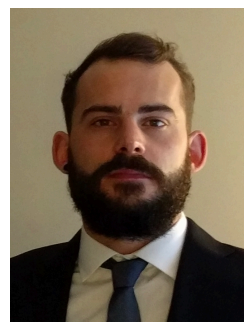

Luca Barbierato (S18) received his M.Sc. degree in Telecommunication Engineering from the University of Brescia, Brescia, Italy, in 2013.

He was a collaborator of SWARM Joint Open Lab of Telecom Italia at Politecnico di Torino, Turin, Italy. In 2016, he joined the Department of Computer and Control Engineering at Politecnico di Torino as a Research Assistant. In 2018, he joined as Ph.D. Candidate the Energy Center LAB, an interdisciplinary project of Politecnico di Torino. His main research interests are energy efficiency and smart cities with particular attention to the design of IoT platforms for energy metering, cosimulation platform for multi-energy system assessment and energy efficiency control strategies.

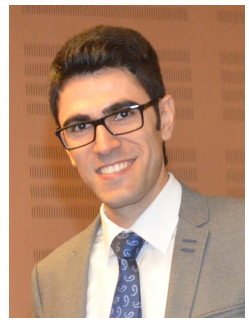

Abouzar Estebsari (M18) received his $\mathrm{PhD}$ in electrical engineering from Department of Energy at Politecnico di Torino, Turin, Italy, in 2015.

He joined the Institute for Automation of Complex Power Systems (ACS) in RWTH Aachen University, Aachen, Germany, as a visiting scientist for a short period in 2016. He is currently an Assistant Professor of power systems in the Department of Energy at Politecnico di Torino. His main research interests include power system security of supply, time co-simulation of power systems. ICT-enabled new services in smart grids, and real-

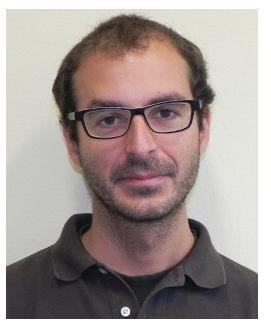

Enrico Pons (M'15) received the master degree in electrical engineering in 2004 and the Ph.D. in industrial safety and risk analysis in 2008 from Politecnico di Torino, Torino, Italy.

$\mathrm{He}$ is currently associate professor at Politecnico di Torino, where he teaches electrical installations. His research activities include complexity in energy systems, power systems security, complex networks methodologies for the analysis of power systems vulnerability, real-time simulation of power systems, integration of renewable generation in distribution networks, smart metering, traction electrification systems and electrical safety. He co-authored more than 70 publications, published in international conferences proceedings and in international journals.

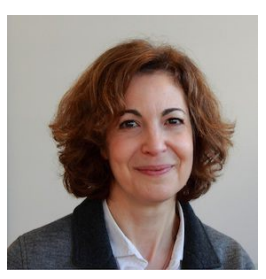

Ferdinanda Ponci (M'00-SM'08) received the $\mathrm{Ph} . \mathrm{D}$. degree in Electrical Engineering from the Politecnico di Milano, Milan, Italy, in 2002.

In 2003, she joined the Department of Electrical Engineering, University of South Carolina, Columbia, SC, USA, as an Assistant Professor, where she became an Associate Professor in 2008. In 2009, she joined the Institute for Automation of Complex Power Systems, E.ON Research Center, RWTH Aachen University, Aachen, Germany, where she is currently a Professor of monitoring and distributed control for power systems. She is involved in research on automation and advanced monitoring of active distribution systems.

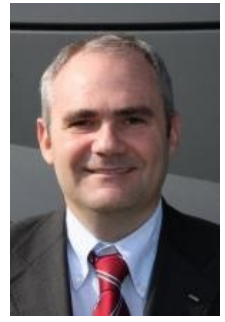

Antonello Monti (M94SM02) received the M.Sc degree (summa cum laude) and the Ph.D. degree in electrical engineering from the Politecnico di Milano, Milan, Italy, in 1989 and 1994 respectively.

He started his career in Ansaldo Industria, Milan, and then moved to the Politecnico di Milano in 1995 as an Assistant Professor. In 2000, he joined the Department of Electrical Engineering, University of South Carolina, Columbia, SC, USA, as an Associate Professor and then a Full Professor. Since 2008, he has been the Director of the Institute for Automation of Complex Power System, E.ON Energy Research Center, RWTH Aachen University, Aachen, Germany. He has authored or co-authored more than 300 peer-reviewed papers published in international Journals and in the proceedings of international conferences.

Dr. Monti is an Associate Editor of the IEEE Systems Journal and IEEE Electrification Magazine. He is a member of the Editorial Board of the Elseviers Journal Sustainable Energy, Grids and Networks and the Founding Board of the Springer Journal Energy Informatics. He was recipient of the 2017 IEEE Innovation in Societal Infrastructure Award. 